\title{
Effects of boron nutrition on arsenic uptake and efflux by rice seedlings
}

\author{
R.L. Zheng ${ }^{1} \&$ G.X. Sun ${ }^{2}$ \\ ${ }^{1}$ Beijing Research \& Development Center for Grasses and Environment, Beijing Academy of Agriculture \\ and Forestry Sciences, Beijing, P.R. China \\ ${ }^{2}$ State Key Laboratory of Urban and Regional Ecology, Research Center for Eco-Environment Sciences, \\ Chinese Academy of Sciences, Beijing, P.R. China
}

\begin{abstract}
The impacts of boron (B) through root application and foliar spray on arsenic (As) uptake and efflux by/in rice seedlings (Oryza sativa L.) were investigated in three hydroponic experiments. Addition of B in culture medium didn't alter concentrations of arsenite $[\mathrm{As}(\mathrm{III})]$, arsenate $[\mathrm{As}(\mathrm{V})]$ and total As in rice seedlings under either As(III) or As(V) exposure. Foliar B supply decreased root As concentrations by $20.9 \%$ under As(V) treatment and by $12.6 \%$ under As(III) treatment, respectively, yet didn't significantly decrease shoot As concentrations. Concentrations of $\mathrm{As}(\mathrm{V})$ were positively related to $\mathrm{B}$ concentration in rice root under $\mathrm{As}(\mathrm{V})$ treatment following foliar B supply $(P<0.05)$. Rice seedlings extruded $105.2 \%$ more As after As(III)-pretreatment than after As $(\mathrm{V})$-pretreatment. Foliar B supply increased the amount of As excreted by As(III)-pretreated rice root by $14.0 \%-16.9 \%(P>0.05)$ and made no effect on As efflux for As(V)-pretreatment seedlings. These results indicate that root application of $\mathrm{B}$ at four times the concentration of As can hardly decrease As accumulation by rice, whereas foliar B supply is conducive to a decline in As acquisition by rice root. It is likely that B channel is at least not the main pathway for $\mathrm{As}(\mathrm{III})$ entering into rice root, and $\mathrm{As}(\mathrm{V})$ distribution mechanism in rice plant may share with B.
\end{abstract}

\section{INTRODUCTION}

Boron (B) mainly occurs in soils or organisms as $\mathrm{B}(\mathrm{OH})_{3}$ which is an uncharged molecule structurally similar to $\mathrm{As}(\mathrm{OH})_{3}$. Many studies have shown that NIP channel proteins such as OsNIP3;1, AtNIP5;1, AtNIP6;1 which facilitate As uptake or transport are also $\mathrm{B}(\mathrm{OH})_{3}$ transporters essential for B uptake by rice or thale cress roots (Hanaoka et al., 2014; Miwa and Fujiwara, 2010). PIP channel proteins (OsPIP2;4 and OsPIP2;7) from rice mediated not only B but also As transport (Kumar et al., 2014). Furthermore, the expression of these aquaporins can be regulated by B level in the environment (Kumar et al., 2014). The tissue specificity of OsNIP3;2 expression is similar to that of B transporter OsNIP3;1 (Chen et al., 2017). It remains unknown whether As uptake and efflux are influenced by $\mathrm{B}$ addition.

\section{METHODS/EXPERIMENTAL}

\subsection{Effect of culture solution B-level on As uptake}

After seedlings of rice were pre-cultured in a full strength nutrient solution for one week, 6 treatments including three B levels $\left(0,10\right.$ and $\left.40 \mu \mathrm{mol} \mathrm{L}^{-1}\right)$ with $10 \mu \mathrm{mol} \mathrm{L}^{-1} \mathrm{As}(\mathrm{III})$ or $\mathrm{As}(\mathrm{V})$ in solution were imposed. Plants were harvested after As exposure for $2 \mathrm{~d}$. Shoot and root were quickly separated, washed with deionized water, blotted dry and ground in a mortar and pestle with liquid $\mathrm{N}$ for further measurements after the fresh weight was recorded.

\subsection{Effect of foliar B supply on As uptake}

Rice seedlings subjected to preculture in a nutrient solution without B for one week were transferred to nutrient solutions containing $10 \mu \mathrm{mol} \mathrm{L}^{-1} \mathrm{As}(\mathrm{III})$ or $\mathrm{As}(\mathrm{V})$ whilst no B addition. Boron was applied through foliage including foliar sprays of deionized water (control), and 9, $36 \mathrm{mmol} \mathrm{L}^{-1} \mathrm{H}_{3} \mathrm{BO}_{3}$ solution. Foliar sprays $(3 \mathrm{~mL}$ per pot) were applied once a day. Plants were harvested as described above after As exposure and foliar B supply for one week. Specially, shoot was rinsed with deionized water containing Triton X-100 three times first to remove B adhering on the surface of leaves.

\subsection{Effect of foliar B supply on As uptake}

Rice seedlings were pre-cultured in a nutrient solution without $\mathrm{B}$ for one week, and $10 \mu \mathrm{mol} \mathrm{L}^{-1} \mathrm{As}$ (III) or $\mathrm{As}(\mathrm{V})$ was added into culture solution afterwards. After As exposure for one week, plant roots were rinsed in an ice-cold desorption solution, and then soaked in the same ice-cold desorption solution for $10 \mathrm{~min}$ to remove apoplastic As. After roots were washed with deionized water, plants were transferred to $50 \mathrm{~mL}$ lightproof centrifuge tubes containing $40 \mathrm{~mL}$ nutrient solution without As and B, and foliar B supply 


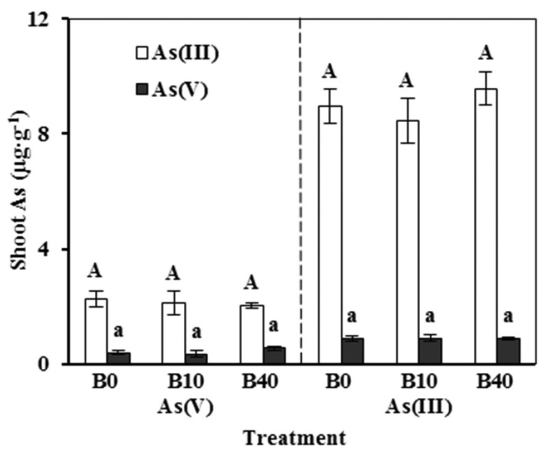

Figure 1. Effects of B level of culture solution on As(III) and $\mathrm{As}(\mathrm{V})$ concentrations in rice shoot.

Table 1. Total As and B concentrations in shoot and root of rice seedlings grown in culture solutions with As (III) or $\mathrm{As}(\mathrm{V})$ under foliar sprays of different B levels.

As Foliar B Shoot As Root As Shoot B Root B species supply $\left(\mu \mathrm{gg}^{-1}\right)\left(\mu \mathrm{gg}^{-1}\right)\left(\mu \mathrm{gg}^{-1}\right)\left(\mu \mathrm{gg}^{-1}\right)$

\begin{tabular}{llrlrl}
\hline & B0 & 7.4 & 27.7 & 4.2 & 2.5 \\
As(V) & B9 & 7.0 & 23.1 & 21.7 & 2.1 \\
& B36 & 6.5 & 21.9 & 70.1 & 1.7 \\
& B0 & 19.2 & 57.2 & 5.8 & 1.1 \\
As(III) & B9 & 19.6 & 60.0 & 24.9 & 1.2 \\
& B36 & 18.2 & 50.0 & 70.4 & 1.1 \\
Significance of & & & & \\
As species & $* * *$ & $* * *$ & ns & $* * *$ \\
Foliar B supply & ns & $*$ & $* * *$ & $*$ \\
As $\times$ B & ns & ns & ns & $*$
\end{tabular}

commenced as described above once plants were transferred. One week later, plants were harvested.

\section{RESULTS AND DISCUSSION}

\subsection{Effect of B level in culture solution on As uptake}

With increasing B concentration in culture solution, up to four times that of As, concentrations of both $\mathrm{As}(\mathrm{III})$ and $\mathrm{As}(\mathrm{V})$ in shoots and roots of rice under either As(III) or As(V) exposure were not changed statistically (Fig. 1). It may be attributed to the highly strict control of the permeability to $\mathrm{B}(\mathrm{OH})_{3}$ by $\mathrm{ar} / \mathrm{R}$ selectivity filter in comparison to As(III).

\subsection{Effects of foliar B supply on As uptake}

Root As concentrations declined significantly by $20.9 \%$ under $\mathrm{As}(\mathrm{V})$ treatment and by $12.6 \%$ under As(III) treatment, respectively $(P<0.05)$, with increasing foliar B level supply (Table 1). However, foliar B supply didn't decrease shoot As concentrations $(P<0.05)$ under either As(V) or As(III) exposure. Root B concentrations decreased by up to $47.1 \%$ $(P<0.05)$ with increasing foliar B levels in $\mathrm{As}(\mathrm{V})$ treatment but not in $\mathrm{As}(\mathrm{III})$ treatment. Root As(V) concentration decreased by up to $29.8 \%$ following foliar

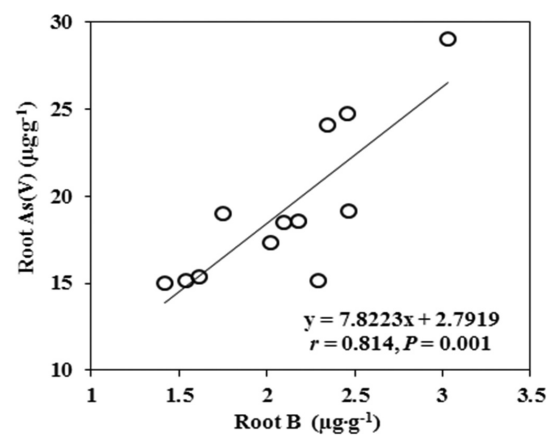

Figure 2. Relationship between root B concentration vs root $\mathrm{As}(\mathrm{V})$ concentration of rice seedlings under foliar sprays of different B levels.

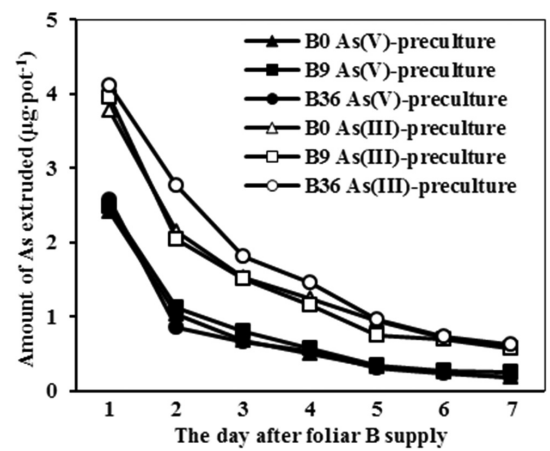

Figure 3. Everyday amount of As excreted into culture solutions by rice seedlings under foliar sprays of $B$.

B supply in $\mathrm{As}(\mathrm{V})$ treatment $(P<0.05)$. Further analysis showed significant positive relationships between root $\mathrm{B}$ concentration versus root $\mathrm{As}(\mathrm{V})$ concentration (Fig. 2) under $\mathrm{As}(\mathrm{V})$ exposure $(P<0.05)$.

\subsection{Effects of foliar B supply on As efflux}

Rice seedlings extruded $105.2 \%$ more As after As(III)pretreatment than after $\mathrm{As}(\mathrm{V})$-pretreatment. Foliar B supply increased the amount of As excreted by As(III)pretreated rice root by $14.0 \%-16.9 \%(P>0.05)$ and made no effect on As efflux for $\mathrm{As}(\mathrm{V})$-pretreatment seedlings (Fig. 3).

\section{CONCLUSIONS}

Boron level in culture solution made no effect on the uptake of As(III) and As(V) by rice roots. Foliar B supply decreased concentrations of total As and $\mathrm{As}(\mathrm{V})$ in root significantly but didn't markedly decrease shoot As concentrations. There was a significant relationship between $\mathrm{B}$ and $\mathrm{As}(\mathrm{V})$ concentrations in rice root under $\mathrm{As}(\mathrm{V})$ exposure following foliar B supply. Foliar B supply didn't increase As efflux under As(III)- or As(V)-preculture treatment. It was likely that B and As $(V)$ share identical regulatory mechanism inside 
rice plant, and $\mathrm{B}$ channels contributed little to As entering into rice roots.

\section{REFERENCES}

Chen, Y., Sun, S.K., Tang, Z., Liu, G.D., Moore, K.L., Maathuis, F.J.M., Miller, A.J., McGrath, S.P. \& Zhao, F.J. 2017. The Nodulin 26-like intrinsic membrane protein OsNIP3;2 is involved in arsenite uptake by lateral roots in rice. J. Exp. Bot. 68(11): 3007-3016.

Hanaoka, H., Uraguchi, S., Takano, J. Tanaka, M. \& Fujiwara T. 2014. OsNIP3;1, a rice boric acid channel, regulates boron distribution and is essential for growth under borondeficient conditions. Plant J. 78(5): 890-902.
Kumar, K., Mosa, K.A., Chhikara, S., Musante, C., White, J.C. \& Dhankher, O.P. 2014. Two rice plasma membrane intrinsic proteins, OsPIP2;4 and OsPIP2;7, are involved in transport and providing tolerance to boron toxicity. Planta 239(1): 187-198.

Miwa, K. \& Fujiwara, T. 2010. Boron transport in plants: coordinated regulation of transporters. Ann. Bot. 105(7): 1103-1108. 